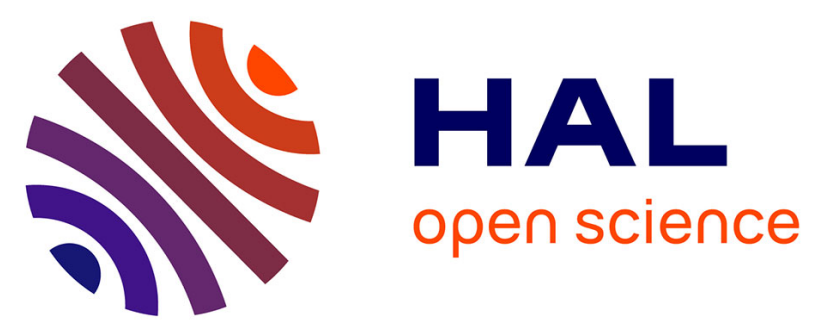

\title{
Rates of Chemical Reactions Embedded in a Metabolic Network by Dissolution Dynamic Nuclear Polarisation NMR
}

Aude Sadet, Emmanuelle M. M. Weber, Aditya Jhajharia, Dennis Kurzbach, Geoffrey Bodenhausen, Emeric Miclet, Daniel Abergel

\section{To cite this version:}

Aude Sadet, Emmanuelle M. M. Weber, Aditya Jhajharia, Dennis Kurzbach, Geoffrey Bodenhausen, et al.. Rates of Chemical Reactions Embedded in a Metabolic Network by Dissolution Dynamic Nuclear Polarisation NMR. Chemistry - A European Journal, 2018, 24 (21), pp.5456-5461. 10.1002/chem.201705520 . hal-02352047

\section{HAL Id: hal-02352047 \\ https://hal.science/hal-02352047}

Submitted on 6 Nov 2019

HAL is a multi-disciplinary open access archive for the deposit and dissemination of scientific research documents, whether they are published or not. The documents may come from teaching and research institutions in France or abroad, or from public or private research centers.
L'archive ouverte pluridisciplinaire HAL, est destinée au dépôt et à la diffusion de documents scientifiques de niveau recherche, publiés ou non, émanant des établissements d'enseignement et de recherche français ou étrangers, des laboratoires publics ou privés. 


\title{
Rates of chemical reactions embedded in a metabolic network by dissolution dynamic nuclear polarization NMR
}

\author{
Aude Sadet, ${ }^{\mathrm{a}, \mathrm{b}}$, Emmanuelle M.M. Weber ${ }^{\mathrm{a}, \mathrm{b}}$, Aditya Jhajharia ${ }^{\mathrm{a}, \mathrm{b}}$, Dennis Kurzbach ${ }^{\mathrm{a}, \mathrm{b}}$, \\ Geoffrey Bodenhausen ${ }^{\mathrm{a}, \mathrm{b}}$, Emeric Miclet ${ }^{\mathrm{a}, \mathrm{b}}$, and Daniel Abergel ${ }^{\mathrm{a}, \mathrm{b},{ }^{*}}$ \\ ${ }^{a}$ Laboratoire des Biomolécules, Département de chimie, Ecole normale supérieure, \\ UPMC Univ. Paris 06, CNRS, PSL Research University, 24 rue Lhomond, 75005 \\ Paris, France \\ ${ }^{\mathrm{b}}$ Sorbonne Universités, UPMC Univ. Paris 06, Ecole normale supérieure, CNRS, \\ Laboratoire des Biomolécules (LBM), 75005 Paris, France
}

January 26, 2018

\begin{abstract}
The isomerisation of 6-phosphogluconolactones and their hydrolyses into 6-phosphogluconic acid form a non enzymatic side cycle of the pentose-phosphate pathway (PPP) in cells. We show that dissolution dynamic nuclear polarization can be used for determining the kinetic rates of the involved transformations in real time. It is found that the hydrolysis of both lactones is significantly slower than the isomeration process, thereby shedding new light onto this subtle chemical process.
\end{abstract}

\section{Introduction}

The study of enzyme kinetics and cell metabolism is central for the understanding of cellular functions and metabolic diseases. The flexibility of enzymatic activity allows the cell to survive despite changing environmental demands by regulating its metabolic pathways. Enzyme dysfunction often leads to severe diseases through the associated alteration of the cellular metabolism. Under various pathological circumstances, reactions that are normally enzymatically driven can be superseded by spontaneous non-enzymatic chemical reactions that may

\footnotetext{
*Electronic address: daniel.abergel@ens.fr
} 

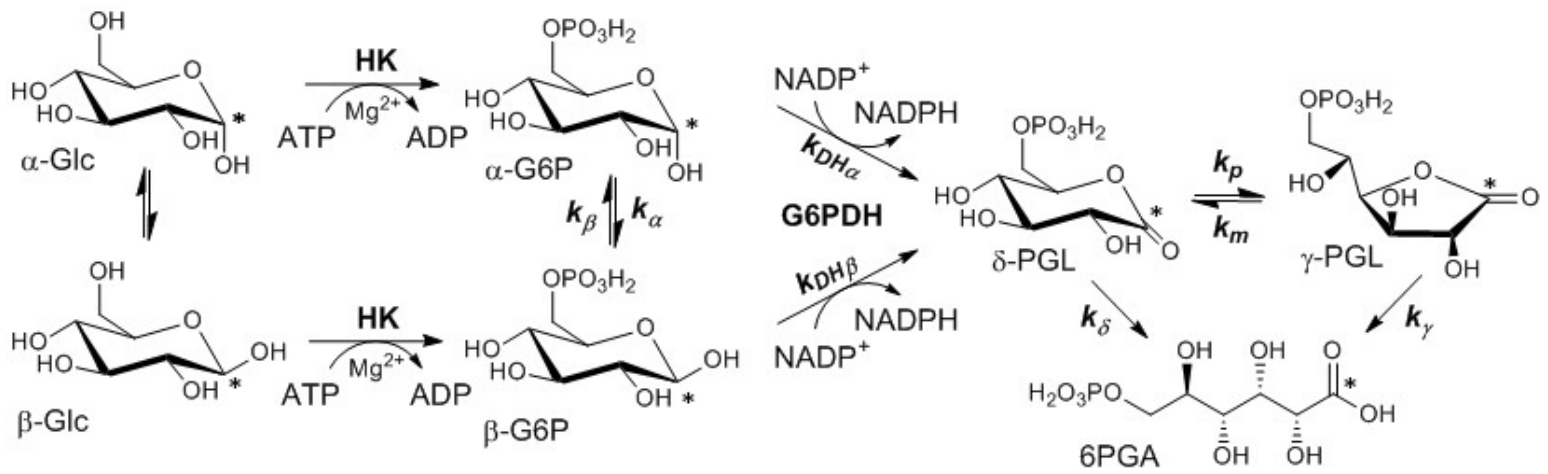

Figure 1: Illustration of the reaction pathway. Phosphorylation of each Glc anomer by Hexokinase (HK) yields the corresponding anomer of G6P. The $\delta$-PGL is produced through G6P oxidation by G6PDH. Subsequent $\delta$-PGL $/ \gamma$-PGL isomerisation is characterized by an equilibrium with forward and backward rates $k_{p}$ and $k_{m}$. Both 6-phosphogluconolactones undergo irreversible hydrolysis with rates $k_{\delta}$ and $k_{\gamma}$. In this work, compounds are uniformly, ${ }^{13} \mathrm{C},{ }^{2} \mathrm{H}$, doubly labeled. $\mathrm{C}_{1}$ atoms are indicated by an asterisk.

generate by-products that are potentially toxic to the cell. [1] The pentose-phosphate pathway (PPP), one of the crucial metabolic pathways in the cell, provides one such example.[2] Once Glucose (Glc) is transformed into Glucose-6-Phosphate (G6P) in the cell, the latter is oxidized into $\delta$-6-Phosphogluconolactone $(\delta$-PGL) through the action of the Glucose-6-Phosphate dehydrogenase $(\mathrm{G} 6 \mathrm{PDH})$. This is the first reaction in the enzymatic cascade of the so-called oxidative stage of the PPP. The natural reaction product of G6PDH, $\delta$-PGL, subsequently undergoes hydrolysis catalyzed by the next enzyme of the pathway, the 6-phosphogluconolactonase (6PGL) to yield 6-phosphogluconic acid (6PGA). In cases of 6PGL deficiency, the $\delta$-PGL accumulates in the cell and partly isomerizes into $\gamma$-PGL.[2, 3] Both lactones can spontaneously hydrolyze to give 6PGA (see Fig.1) but may also react with endogeneous nucleophilic species, as they are highly reactive electrophiles. [4] To investigate this physiologically important series of events that takes place in the context of PPP deficiency, and without the direct intervention of any enzyme, we have used dissolution dynamic nuclear polarization (D-DNP). This technique allows one to boost the nuclear magnetic resonance (NMR) signal intensities of the various metabolites, thereby allowing for the monitoring of the kinetics associated with these reactions in real time. D-DNP allows to overcome the low sensitivity of ${ }^{13} \mathrm{C}$ NMR by providing signal enhancements up to five orders of magnitude for a wide range of hyperpolarized substrates or metabolites. [5, 6, 7, 8] Such a sensitivity enhancement renders the study of chemical reactions on time scales of a few seconds possible by making signal averaging unnecessary. This provides a unique access to potentially rich information, by allowing the simultaneous monitoring of transformation kinetics of several species.

In this communication, we report on the observation of the spontaneous rearrangement of DNP-polarized $\delta$ - and $\gamma$-PGLs and their hydrolysis into 6PGA in solution, on a time window 
of a few tens of seconds. The polarized sample consisted of $50 \mu \mathrm{L}$ of a solution containing $600 \mathrm{mM}$ uniformly, doubly labeled $\left[\mathrm{U}^{13} \mathrm{C},{ }^{2} \mathrm{H}\right]$-Glc in a $90 \%$ deuterated water/ethylene glycol mixture. TEMPOL (4-hydroxy-2,2,6,6-tetramethylpiperidine-1-oxyl) at a concentration of 50 $\mathrm{mM}$ was used as the paramagnetic polarizing agent. This solution was inserted in a Bruker prototype D-DNP apparatus operating at $B_{0}=6.7 \mathrm{~T}$ and at $T=1.2 \mathrm{~K}$. By employing ${ }^{1} \mathrm{H}-$ $\rightarrow{ }^{13} \mathrm{C}$ cross-polarization[9] a ${ }^{13} \mathrm{C}$ polarization of $\mathrm{P}\left({ }^{13} \mathrm{C}\right)=49 \%$ was achieved in the solid state. When the polarization reached its maximum, the sample was dissolved by fast injection of $5 \mathrm{~mL} \mathrm{D}_{2} \mathrm{O}$ overheated to $180^{\circ} \mathrm{C}$ at 10.5 bar into the frozen sample. The sample was subsequently transferred to a $9.4 \mathrm{~T}(400 \mathrm{MHz})$ detection NMR spectrometer using a continuous flow of He at 8 bars during $6 \mathrm{~s}$. During this delay, the hyperpolarized sample was degassed in a phase separation compartment located inside the NMR magnet bore. The hyperpolarized Glc solution was then injected into an NMR tube containing $2 \mathrm{~mL}$ of a solution of a reactive medium containing the relevant enzymes and their cofactors, which has been waiting at $309 \mathrm{~K}$ (see Fig. 2). The injection was completed within $10 \mathrm{~s}$. The beginning of the data acquisition $(t=0)$ was synchronized with the start of the injection. Since only $2.6 \mathrm{~mL}$ of the solution were injected into the NMR tube, $15 \mu$ moles of glucose actually reached the reactive medium, which amounts to a concentration of ca. $3 \mathrm{mM}$ in a total reaction volume of $4.6 \mathrm{~mL}$. Finally, the chemical reactions were monitored by recording ${ }^{13} \mathrm{C}-\mathrm{NMR}$ free induction decays at $1 \mathrm{~s}$ second intervals, using $10^{\circ}$ flip angle detection pulses. Signal enhancement, estimated at $t=5 \mathrm{~s}$ after injection, was 4100 over the entire ${ }^{13} \mathrm{C}$ spectrum (in the presence of enzymes), corresponding to a ${ }^{13} \mathrm{C}$ polarization of $\sim 3.3 \%$.

The enzyme quantities in the $2 \mathrm{~mL}$ solution waiting in the NMR tube prior to injection were adjusted to allow both the phosphorylation of the $\sim 15 \mu$ moles of Glc, and the subsequent G6P oxidation at a rate of $\sim 10 \mu \mathrm{mol} . \mathrm{s}^{-1}$, in order to ensure complete conversion of the injected hyperpolarized Glc within a few seconds, i.e., while the hyperpolarization is still high enough. One unit of enzyme activity converts $1.0 \mu$ mole of substrate per minute, so that the relevant enzyme masses were readily calculated from their specific activities (i.e., units per mg of enzyme, as given by the supplier). The values used in the experiment are given in the caption of Fig. 2. The initial goal was to obtain, after a few seconds, a solution containing only $\delta$-PGL, and its two reaction products $\gamma$-PGL and 6PGA. Note that the choice of the temperature $\mathrm{T}=309 \mathrm{~K}$ in the NMR spectrometer was made in order to achieve longer ${ }^{13} \mathrm{C} T_{1}$ relaxation times and a prolonged time window for NMR detection $\left(T_{1} \sim 15 \mathrm{~s}\right.$ for $\left[\mathrm{U}^{13} \mathrm{C},{ }^{2} \mathrm{H}\right]$-Glc at $309 \mathrm{~K}$ in pure $\mathrm{D}_{2} \mathrm{O}$ - see Supporting Information), whilst maintaining the enzymes sufficiently active despite the fact that they may not be operating at their presumably optimum temperatures. In this study, we used enzyme concentrations that were similar to the typical high values that can be found in cells, in the range $0.1 \mu \mathrm{M}$ to $100 \mu \mathrm{M}$, especially when enzymatic pathways are confined in organelles. In some cases, enzyme concentrations can even exceed metabolite concentrations.[10, 11] In D-DNP experiments, the use of labeled compounds is widespread. ${ }^{13} \mathrm{C}$-labelled molecules are commonly used to increase the signal with respect to the one obtained with natural abundance compounds (for a recent review, see 


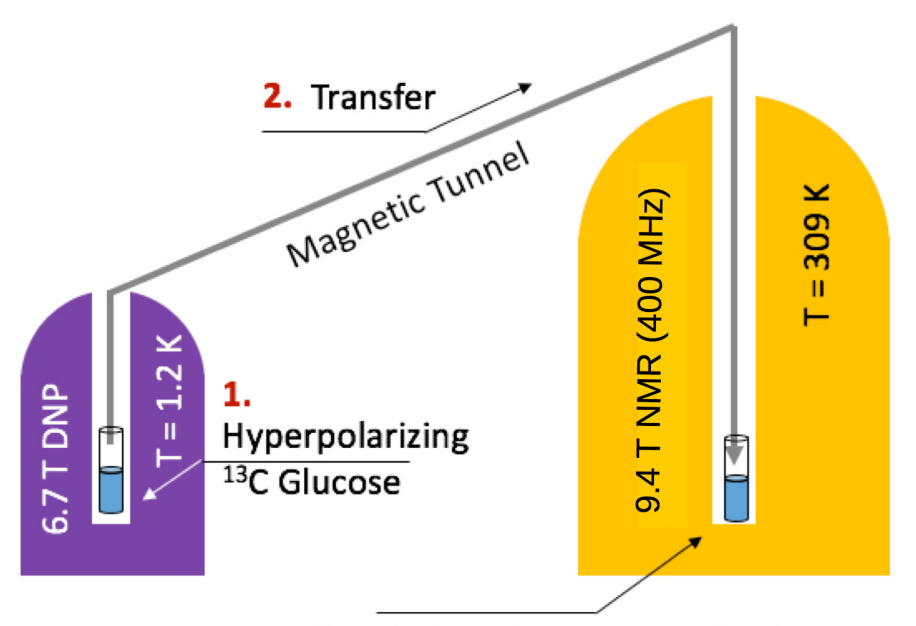

\section{Solution of enzymes and cofactors}

Figure 2: After polarizing $\left[\mathrm{U}_{-}^{13} \mathrm{C},{ }^{2} \mathrm{H}\right]$-Glc at $1.2 \mathrm{~K}$ and $6.7 \mathrm{~T}$ the sample is transferred to a detection spectrometer operating at $9.4 \mathrm{~T}$ to observe the metabolic conversion of the hyperpolarized substrate. The quick conversion of Glc into $\gamma$-PGL has been achieved using the following conditions: 6 mg of yeast Hexokinase $(28 \mu \mathrm{M}$ corresponding to $\approx 600 \mathrm{U})$ and $1.4 \mathrm{mg}$ of $\mathrm{G} 6 \mathrm{PDH}(5.5 \mu \mathrm{M}$ corresponding to $\approx 300 \mathrm{U})$ together with the cofactors ATP $(33 \mathrm{mM})$ and $\mathrm{NADP}^{+}(30 \mathrm{mM})$ in $60 \mathrm{mM}$ HEPES buffer, $\mathrm{pH}=7.8,30 \mathrm{mM} \mathrm{MgCl}_{2}, 309 \mathrm{~K}$.

for instance Ref[12] and references therein). When possible, the observation of quaternary carbons is preferred in order to take advantage of $T_{1}$ relaxation times that are longer than the ones of proton-coupled carbons.[13] Alternatively, ${ }^{13} \mathrm{C},{ }^{2} \mathrm{H}$ doubly labeled compounds may be used to extend the ${ }^{13} \mathrm{C}$ T1 relaxation times of methylene $[7,14]$ or methine groups (as in the case of glucose) $[15,16,17,18,19,20,21]$ Note that deuteration may in principle affect kinetics - a fact that should be considered for virtually all dissolution DNP experiments based on such substrates. $\mathrm{D}_{2} 0$, which is used as the dissolution solvent, may also be used to increase $T_{1}$, and in this work, NMR experiments were performed in a $\mathrm{H}_{2} 0 / \mathrm{D}_{2} 0$ mixture $(43 / 57 \mathrm{v} / \mathrm{v})$, which may affect the kinetics of enzymes that normally operate in pure water. However, the goal of this work was to investigate a set of chemical reactions that are not catalyzed by the enzymes present in the reactive medium. Yet, some effect of the presence of $\mathrm{D}_{2} 0$ in the solution on the kinetics of the $\delta / \gamma$-PGL pathway may not be ruled out.

The characteristic ${ }^{13} \mathrm{C}_{1}$ signals of the $\alpha$ and $\beta$ anomers of uniformly labeled $\left[\mathrm{U}_{-}{ }^{13} \mathrm{C},{ }^{2} \mathrm{H}\right]$-Glc in solution appear at 92.9 and $96.6 \mathrm{ppm}$ (see Fig. 3). The ${ }^{2} \mathrm{H}$ decoupled spectra exhibit doublets due to ${ }^{1} J_{\mathrm{C}_{1} \mathrm{C}_{2}}$ couplings, whilst the smaller couplings $\left({ }^{2} J_{\mathrm{C}_{1} \mathrm{C}_{3}}\right.$ and ${ }^{3} J_{\mathrm{C}_{1} \mathrm{C}_{6}}$ for $\beta ;{ }^{2} J_{\mathrm{C}_{1} \mathrm{C}_{5}}$ and ${ }^{3} J_{\mathrm{C}_{1} \mathrm{C}_{6}}$ for $\alpha$ ) remain unresolved.[8] The decay of the Glc resonances observed after injection is due to the combination of the loss of substrate caused by enzymatic phosphorylation, and to spin-lattice relaxation that drives the initial hyperpolarization towards thermal equilibrium. The ${ }^{13} \mathrm{C}_{1}$ signals of both $\alpha$ and $\beta$ anomers of Glc completely disappear at times larger than 
$\sim 9 \mathrm{~s}(t=0$ corresponds to the beginning of the injection, which starts $6 \mathrm{~s}$ after dissolution and is completed within $10 \mathrm{~s}$ ), which attests for the fast transformation of Glc into G6P by hexokinase. At short times (4 s after initiation of the injection), the characteristic ${ }^{13} \mathrm{C}_{1}$ doublets of G6P build up as a result of Glc phosphorylation, and appear $\sim 0.2 \mathrm{ppm}$ downfield with respect to their Glc counterparts. In the case of the $\alpha$ anomer, the doublet remains observable until $t \approx 20 \mathrm{~s}$. In contrast, the evolution of the $\beta$-G6P resonances is strikingly different. At $t=4 \mathrm{~s}$ after the beginning of the injection, only a small $\beta$-G6P signal is present (see Fig. 3), which becomes undetectable for $t \geq 9 \mathrm{~s}$. These observations indicate that the transformation rate of the $\beta$ anomer of G6P by G6PDH is significantly faster than the oxidation of its $\alpha$ anomer (assuming that the $T_{1}$ relaxation times of ${ }^{13} \mathrm{C}_{1}$ in both the $\alpha$ and $\beta$-G6P anomers are similar [8]), and therefore provide evidence of the $\beta$-anomeric selectivity of yeast G6PDH, implying that $\beta$-G6P is the preferred substrate of G6PDH. Salas et al.[22] have demonstrated experimentally that Glc is a very low affinity substrate of G6PDH, which acts with high selectivity towards its $\beta$-anomer. However, the authors solely inferred from these results that the same $\beta$-selectivity can be assumed for G6P. We have performed control experiments that allow one to estimate the catalytic effect of G6PDH on Glc.We have found that the rate of catalysis is orders of magnitude slower than the rate of oxidation of G6P by G6PDH. The former can therefore be safely discarded on time scales relevant to our DDNP experiments (see Supplementary Information). To our knowledge, we present the first direct experimental evidence of $\beta$-G6P selectivity of yeast G6PDH. The influence of the $\alpha / \beta$ mutarotation of G6P should also be considered. Indeed, it has been $\operatorname{shown}[23,24,25]$ that this process takes place on a much faster timescale for Glu than for G6P; up to a factor of $\sim 200$. For Glu, the anomerization timescale is on the order of minutes at $309 \mathrm{~K}$, whereas for G6P, it is likely on the order of seconds, in other words, on the timescales of the kinetic processes observed in the experiments presented here. This is important in the case at hand, since $\alpha$-G6P is detected until $t \sim 20 \mathrm{~s}$, and the kinetics of mutarotation may superimpose with the observed kinetics of the isomerisation/hydrolysis of the PGLs. It is therefore necessary to include the enzymatic oxidation of $\mathrm{G} 6 \mathrm{P}$ in the kinetics analysis of the lactone pathway.

G6P is subsequently transformed into $\delta$-PGL. The ${ }^{13} \mathrm{C}_{1}$ lactone signals appear in the $174-179$ ppm range. At $t=4 \mathrm{~s}$, the presence of the characteristic $\delta$-PGL signal at $175 \mathrm{ppm}$ confirms that the enzyme quantities used are sufficient to rapidly produce the precursor of the isomerisation/hydrolysis pathway. Moreover, for $t>9 \mathrm{~s}$, additional weak signals appear at 178.1 and $179.8 \mathrm{ppm}$, corresponding to $\gamma$-PGL and 6PGA, respectively. These observations provide direct evidence for the spontaneous hydrolysis of the PGLs into 6PGA.

The time-dependencies of the signal intensities of all species are depicted in Fig. 4. Interestingly, a substantial amount of $\gamma$-PGL is already produced at $t=10 \mathrm{~s}$, leading to lactone signals that are both more intense than those of 6 PGA, which is a qualitative indication that the $\delta$ - to $\gamma$-PGL conversion is faster than the spontaneous hydrolysis of either lactones, assuming similar values for $T_{1}$. The signal-to-noise ratio (SNR) of the hyperpolarized $\delta$-PGL signal at $t=10 \mathrm{~s}$ was ca. 600 , an order of magnitude larger than the signal of its $\gamma$-PGL coun- 


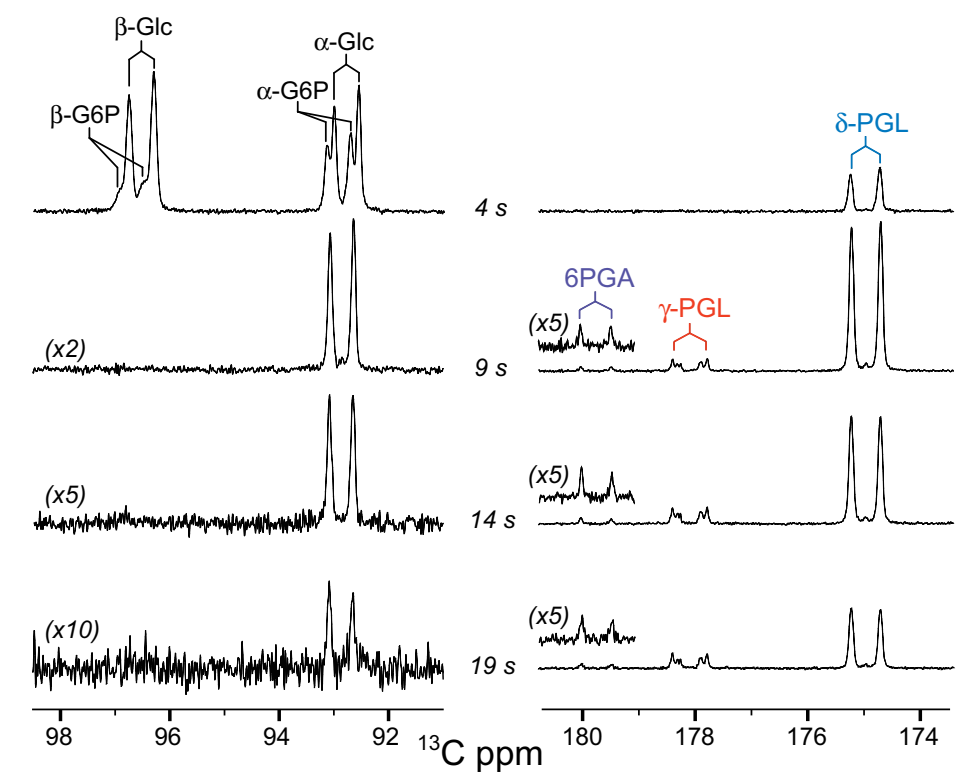

Figure 3: ${ }^{13} \mathrm{C}$ spectra obtained after injection of DNP-polarized Glc. (Left) For $t=4 \mathrm{~s}$, both polarized $\alpha / \beta$ anomers of $\mathrm{G} 6 \mathrm{P}$ are present in the spectrum, at a typical $\sim 0.2 \mathrm{ppm}$ downfield shift with respect to Glc. Moreover, complete signal clearance of both $\alpha / \beta$ anomers of Glc is observed after $t=9 \mathrm{~s}$. In addition, $\beta$-G6P is also rapidly consumed and its signal has totally disappeared after $t \geq 9$ $\mathrm{s}$, whereas $\alpha$-G6P is not fully oxidized. These spectra illustrate the high selectivity of G6PDH that acts essentially on the $\beta$-anomer. (Right) $\delta$-PGL is present at $t=4 \mathrm{~s}$. When $\beta$-G6P oxidation is complete $(t \geq 9 \mathrm{~s})$, a non negligible fraction is already transformed into $\gamma$-PGL, and a small 6PGA signal is already detectable (in all cases, $t=0$ refers to the start of the injection, which has completed at $t=10$ s).

terpart, and two orders of magnitude larger than the signal of 6PGA, which always remained low, below $\sim 5$, although clearly detectable.

A quantitative analysis of the time series of NMR peak intensities obtained after complete injection was performed based on a first order kinetic model, as introduced in Ref.[2] (cf. Fig. 1 ). From the above, the Glc signals are discarded from the kinetic analysis of this reaction network (the signals have totally disappeared at $t \geq 10 \mathrm{~s}$ ). However, oxidation and mutarotation of G6P anomers are included. Only the kinetic constants relative to the $\delta / \gamma$-PGL isomerisation and hydrolysis are extracted, and these are disentagled from all parameters pertaining to enzyme kinetics proper, as shown in the following. The system of differential equations that describes the evolution of the $\mathrm{z}$ components $\mathbf{M}$ of the ${ }^{13} \mathrm{C}$ magnetizations at the $\mathrm{C}_{1}$ sites, in $\alpha$ - and $\beta$-G6P, in $\delta / \gamma$-PGLs and 6PGA, between two consecutive signal acquisitions, writes 

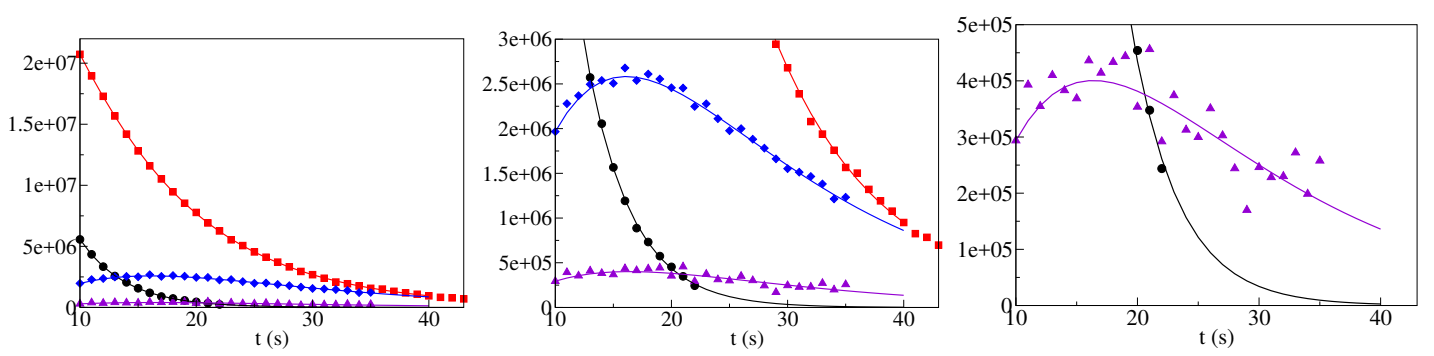

Figure 4: Buildup and decay curves of the $\delta$-PGL (red), $\gamma$-PGL (blue), 6PGA (purple) and $\alpha$-G6P (black) NMR signal intensities upon the chemical reaction cycle. Dots correspond to experimental values. Solid lines indicate fits to the model of Eqs. 1-2 (see text). The time axis starts at $t=10 \mathrm{~s}$, which corresponds to the first point of the kinetics analysis.

$\frac{d}{d t} \mathbf{M}=\mathbf{A} \mathbf{M}$. In this expression, $\mathbf{M}=\left[M_{\alpha}, M_{\beta}, M_{\delta}, M_{\gamma}, M_{A}\right]^{t}$, and:

$$
\mathbf{A}=\left[\begin{array}{ccccc}
-\left(k_{\alpha}+k_{\mathrm{DH} \alpha}+R_{1 \alpha}\right) & k_{\beta} & 0 & 0 & 0 \\
k_{\alpha} & -\left(k_{\beta}+k_{\mathrm{DH} \beta}+R_{1 \beta}\right) & 0 & 0 & 0 \\
k_{\mathrm{DH} \alpha} & k_{\mathrm{DH} \beta} & -\left(k_{p}+k_{\delta}+R_{1 \delta}\right) & k_{m} & 0 \\
0 & 0 & k_{p} & -\left(k_{m}+k_{\gamma}+R_{1 \gamma}\right) & 0 \\
0 & 0 & k_{\delta} & k_{\gamma} & -R_{1 A}
\end{array}\right]
$$

where $R_{1 \alpha}, R_{1 \beta}, R_{1 \delta}, R_{1 \gamma}, R_{1 A}$ are the longitudinal relaxation rates of $\delta$-PGL, $\gamma$-PGL and 6 PGA, respectively. The rate constants $k_{x}$ associated to the various reactions are indicated in the reaction scheme of Fig. 1. Therefore, the signal $S(n \Delta t)=\left[S_{\alpha}, S_{\beta}, S_{\delta}, S_{\gamma}, S_{A}\right]^{t}$ observed after the $\mathrm{n}$-th pulse with flip angle $\theta$ is:

$$
S(n \Delta t)=\exp (\mathbf{A} n \Delta t) \cos ^{n} \theta S(0),
$$

where $\Delta t$ is the time interval between two consecutive pulses and the initial value of the signal at time $t_{0}=10 \mathrm{~s}$ is $S(0)=\left[S_{\alpha}\left(t_{0}\right), S_{\beta}\left(t_{0}\right), S_{\delta}\left(t_{0}\right), S_{\gamma}\left(t_{0}\right), S_{A}\left(t_{0}\right)\right]^{t}$. Similar first-order kinetics models have been used to describe consecutive reactions by DDNP.[7] In the case at hand, the signal of the $\beta$ anomer of G6P vanishes for $t \geq 10 \mathrm{~s}$. This justifies the quasi-steady-state assumption, where $\beta$-G6P is consumed much faster than produced from the $\alpha$ anomer of G6P through mutarotation. Therefore, $\left[G 6 P_{\beta}\right]$ is so low as to justify the approximations $\left[G 6 P_{\beta}\right] \approx 0$ and $\frac{d\left[G 6 P_{\beta}\right]}{d t}=0$. These approximations imply that:

$$
\frac{d S_{\alpha}}{d t} \approx-\left(k_{\alpha}+k_{\mathrm{DH} \alpha}+R_{1 \alpha}\right) S_{\alpha}
$$


and:

$$
\frac{d S_{\beta}}{d t}=k_{\alpha} S_{\alpha}-\left(k_{\beta}+k_{\mathrm{DH} \beta}+R_{1 \beta}\right) S_{\beta} \approx 0
$$

which implies:

$$
\frac{d S_{\delta}}{d t}=\left(k_{\mathrm{DH} \alpha}+\frac{k_{\mathrm{DH} \beta} k_{\alpha}}{k_{\mathrm{DH} \beta}+k_{\beta}+R_{1 \beta}}\right) S_{\alpha}-\left(k_{p}+k_{\delta}+R_{1 \delta}\right) S_{\delta}+k_{m} S_{\delta}
$$

Introducing $k=k_{\mathrm{DH} \alpha}+\frac{k_{\mathrm{DH} \beta} k_{\alpha}}{k_{\mathrm{DH} \beta}+k_{\beta}+R_{1 \beta}}$, and $k^{\prime}=k_{\alpha}+k_{\mathrm{DH} \alpha}+R_{1 \alpha}$, the differental system reduces to:

$$
\begin{aligned}
\frac{d S_{\alpha}}{d t} & =-k^{\prime} S_{\alpha} \\
\frac{d S_{\delta}}{d t} & =k S_{\alpha}-\left(k_{p}+k_{\delta}+R_{1 \delta}\right) S_{\delta}+k_{m} S_{\gamma} \\
\frac{d S_{\gamma}}{d t} & =k_{p} S_{\delta}-\left(k_{m}+k_{\gamma}+R_{1 \gamma}\right) S_{\gamma} \\
\frac{d S_{\mathrm{A}}}{d t} & =k_{\delta} S_{\delta}+k_{\gamma} S_{\gamma}-R_{1 \mathrm{~A}} S_{\mathrm{A}}
\end{aligned}
$$

Through the quasi-steady state approximation, the large number of parameters (eight rate constants, five relaxation parameters and five initial signal amplitudes at $t_{0}=10 \mathrm{~s}$ ) can be reduced, with the use of the lumped parameters $k$ and $k^{\prime}$, and despite its complexity, this model reduction allows for the determination of the kinetics constants that characterize $\delta$ - $/ \gamma$-PGL isomerisation and hydrolysis, the parameters of interest in this study. Here, the upstream processes (mutarotation and enzymatic reactions) merely appear as a "source term" of the $\delta$-PGL signal.

Fitting was performed through a homemade Scilab,[26] routine implementing the differential evolution algorithm[27, 28] and the strategy described in Ref.[8]. This approach is useful when the number of parameters is large, and fitting procedures that rely on derivatives tend to get trapped in local minima. Due to very high signal intensities in D-DNP experiments, the main source of experimental errors is not the noise of the detection circuit, an approximately Gaussian noise process, but systematic errors and uncontrolled events that may occur during the injection process. This prevents from obtaining statistical estimates of the parameter uncertainties (which would require to repeat the same experiment many times. [8] In the experiments described here, only $\delta$-PGL signals have very high SNRs. In contrast, for signals with lower intensities, $\alpha$-G6P, $\gamma$-PGL and 6PGA, a Gaussian noise assumption may be relevant. A Monte Carlo (MC) simulation was used to compute parameter histograms from pseudo-experimental noise to determine the influence of the latter on the model parameters. However, as the Gaussian noise assumption does not hold for $\delta$-PGL, the statistical $\chi^{2}$ criterion does not apply. Fitting results are depicted in Fig. 5. The parameters $k_{p}, k_{m}, k_{\delta}$ and $R_{1 \delta}$ exhibit monomodal and symmetric distributions, in contrast to the distributions of $k_{\gamma}, R_{1 \gamma}$ and $R_{1 A}$. The latter are asymmetric, featuring two peaks, and include nonrealistic values. Moreover, a number of $R_{1 \gamma}$ rates that are too low to be physically meaningful were also discarded. Finally, a peak around $\sim 0.5 \mathrm{~s}^{-1}$ in the distribution of $R_{1 A}$ did not seem compatible with the expected 
relaxation times of a carbonyl ${ }^{13} \mathrm{C}$ nucleus of such a small molecule. To improve the distributions of the parameter values, an experimental value $R_{1 \text { Aexp }}$, measured in a similar reaction medium (see Supporting Information), was added as an experimental constraint. Use of this prior knowledge resulted in narrower distributions of the remaining parameters and in the disappearance of double-peaked histograms, without affecting the distributions of the forward and backward isomerization rates, $k_{p}$ and $k_{m}$ (see Fig. 5). The analysis was also performed using the flip angle $\theta$ as an additional adjustable parameter, and/or by starting the fitting procedure at $t_{0}=11,12$ or $13 \mathrm{~s}$. None of these tests altered the conclusions. The means and rmsds of the model parameters obtained are summarized in Table 1.

Table 1: Parameter estimates from the distributions of Fig. 5, either leaving $R_{1 A}$ a free parameter or using the measured value $R_{1 \text { Aexp }}$ as a constraint.

\begin{tabular}{lllll}
\hline & $\begin{array}{l}R_{1 A} \text { measured } \\
\text { mean }\left(\mathrm{s}^{-1}\right)\end{array}$ & $\sigma\left(\mathrm{s}^{-1}\right)$ & $\begin{array}{l}R_{1 A} \text { free } \\
\text { mean }\left(\mathrm{s}^{-1}\right)\end{array}$ & $\sigma\left(\mathrm{s}^{-1}\right)$ \\
\hline$k_{p}$ & $19.8 \times 10^{-3}$ & $6 \times 10^{-4}$ & $19.8 \times 10^{-3}$ & $6 \times 10^{-4}$ \\
$k_{m}$ & $12.2 \times 10^{-3}$ & $5.5 \times 10^{-3}$ & $12.0 \times 10^{-3}$ & $5.3 \times 10^{-3}$ \\
$k_{\delta}$ & $3.1 \times 10^{-3}$ & $7 \times 10^{-4}$ & $3.0 \times 10^{-3}$ & $1 \times 10^{-3}$ \\
$k_{\gamma}$ & $5.9 \times 10^{-3}$ & $2.4 \times 10^{-3}$ & $3.4 \times 10^{-3}$ & $2.4 \times 10^{-3}$ \\
$R_{1 \delta}$ & $75 \times 10^{-3}$ & $3 \times 10^{-3}$ & $75 \times 10^{-3}$ & $3 \times 10^{-3}$ \\
$R_{1 \gamma}$ & $55 \times 10^{-3}$ & $6 \times 10^{-3}$ & $27 \times 10^{-3}$ & $6 \times 10^{-3}$ \\
$R_{1 A}$ & 0.110 & 0.008 & $293 \times 10^{-3}$ & $184 \times 10^{-3}$ \\
$k$ & 0.086 & 0.012 & 0.086 & 0.012 \\
$k^{\prime}$ & 0.24 & 0.02 & 0.25 & 0.02 \\
\hline
\end{tabular}

The experiments presented here exemplify, in a biological context, the power of DDNP experiments to monitor chemical reactions that take place on timescales of seconds. Moreover, the analysis of the kinetics, based on the reaction pathway depicted in Fig. 1, leads to several interesting conclusions. Our results indicate that PGL hydrolysis is irreversible, which is attested by the absence of remaining $\delta$ - and $\gamma$-PGL in the reactive medium. In addition, the forward and backward isomerisation rates are found to have a ratio $k_{p} / k_{m} \sim 1.6$. The hydrolysis rate constants of both isomers have a ratio $k_{\delta} / k_{\gamma} \sim 0.5$, both being significantly smaller than the isomerisation rates. These results can be compared to previous investigation performed under significantly different conditions $(\mathrm{T}=278 \mathrm{~K}$ and $\mathrm{pH} 6.7)$, [2] where kinetics takes place on a timescale of hours, rather than seconds. In that case, the ratio of forward to backward isomerisation rates was $\sim 1$.3. Furthermore, under these experimental conditions, the kinetic constant of $\gamma$-PGL hydrolysis was $k_{\gamma} \sim 0$, suggesting that this compound accumulates in the absence of 6PGL that normally catalyses the hydrolysis of $\delta$-PGL into 6PGA. This confirms the critical dependence of these spontaneous rearrangements on environmental conditions. In the present study, the hydrolysis rates of both lactones are 2-7 times slower than their isomerisation rates. This finding supports the assumption that in the absence of the lactonase (6PGL) both species may accumulate and cause damage to the cell through their potent electrophilic properties.[29]

The analysis of the results presented in this work is also consistent with known facts about the mutarotation kinetics of G6P. Based on our measurements of $R_{1 \alpha}$ performed on $\left[\mathrm{U}_{-}{ }^{13} \mathrm{C},{ }^{2} \mathrm{H}\right]$ Glc in pure $\mathrm{H}_{2} \mathrm{O}$ and pure $\mathrm{D}_{2} \mathrm{O}$ at $309 \mathrm{~K}$ (Supplementary Information), and again assuming 

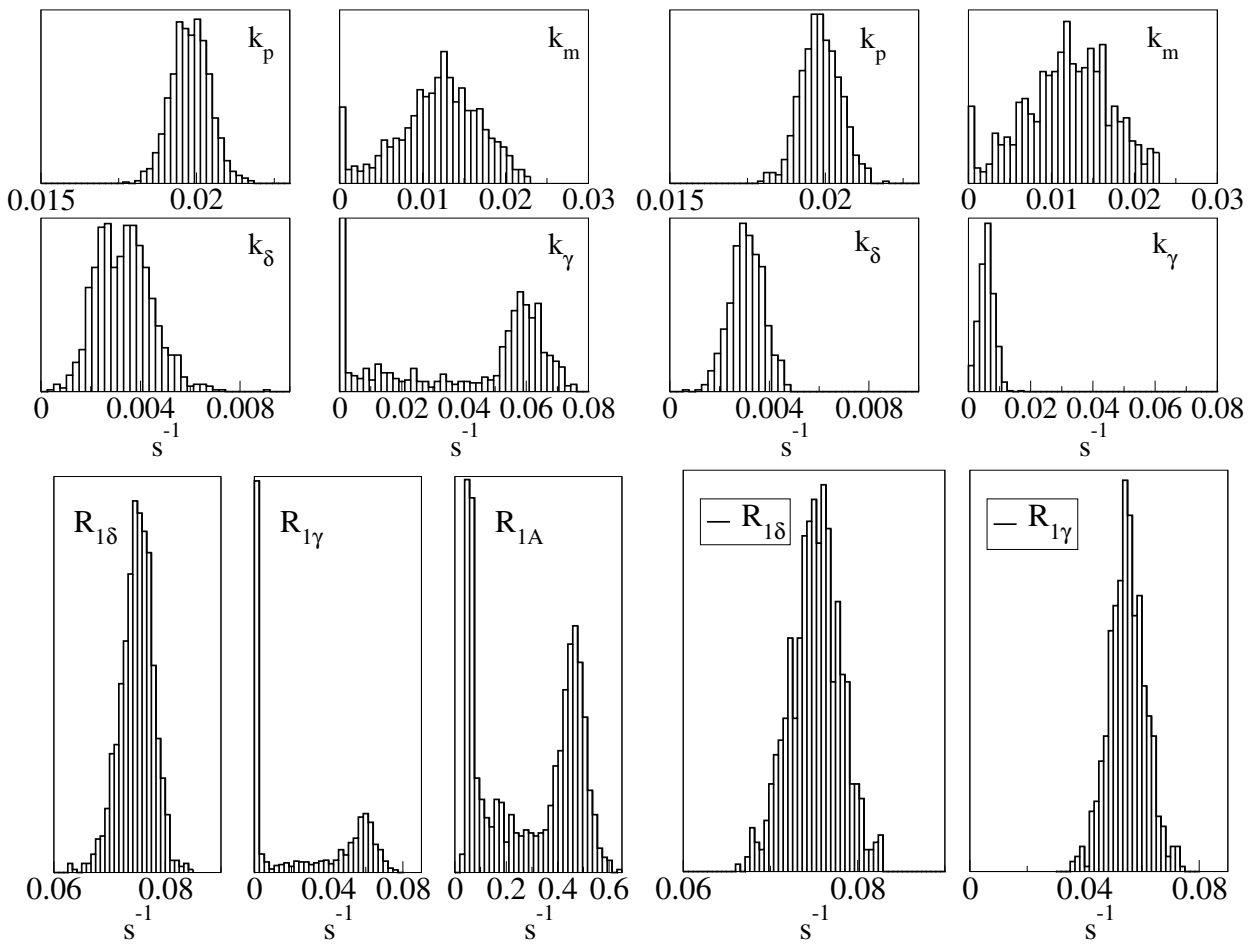

Figure 5: Parameter histograms obtained from $1200 \mathrm{MC}$ iterations using (left panels) all free parameters or (right panels) the experimental value $R_{1 A \text { exp }}=0.110 \pm 0.008 \mathrm{~s}^{-1}$ measured on doubly-labelled 6PGA (Supporting Information). When $R_{1 A, \exp }$ was used $R_{1 A \text {,exp }}$ was drawn from a normal distribution $\mathcal{N}\left(R_{1 A, \exp }, \sigma_{1 A, \exp }\right)$.

that the ${ }^{13} \mathrm{C}_{1} T_{1}$ relaxation times of the anomeric carbons in Glc and $\mathrm{G} 6 \mathrm{P}$ are relatively similar, [8] the relaxation rate $R_{1 \alpha}$ would be on the order of $\sim 0.07 \mathrm{~s}^{-1}$. Therefore, with the additional assumption that $k_{\mathrm{DH} \alpha}$ is negligible (i.e, $\beta$ selectivity of G6PDH), and using the value $\left\langle k^{\prime}\right\rangle \sim 0.24 \mathrm{~s}^{-1}$, a rough estimate of $k_{\alpha} \approx k^{\prime}-R_{1 \alpha} \approx 0.17 \mathrm{~s}^{-1}$ is found. Besides, we have obtained a value of $K=\frac{k_{\alpha}}{k_{\beta}} \approx 1.7$ for the $\alpha / \beta \mathrm{G} 6 \mathrm{P}$ equilibrium constant at $309 \mathrm{~K}$ (Supporting Information), which leads to a crude estimate $k_{\beta} \approx 0.1 \mathrm{~s}^{-1}$. In a previous study,[25] the values $k_{\alpha} \sim 0.06 \mathrm{~s}^{-1}$ and $k_{\beta} \sim 0.04 \mathrm{~s}^{-1}$ were experimentally determined at $298 \mathrm{~K}$. Then, using the activation energy derived in Ref. [24] and assuming an Arrhenius law, this value can be estimated as $k_{\alpha} \sim 0.24 \mathrm{~s}^{-1}\left(k_{\beta} \sim 0.14 \mathrm{~s}^{-1}\right)$ at $309 \mathrm{~K}$, in consistency with our calculations and with our measured $\alpha / \beta \mathrm{G} 6 \mathrm{P}$ equilibrium constant.

To our knowledge, hardly any work on the isomerisation and hydrolysis of 6 -phosphogluconolactones has been published so far. However, some studies have focused on the $\delta$ - $/ \gamma$-gluconolactone (GL) isomerisation and hydrolysis reaction cycle, which obviously bears some strong analogy with the one studied here (see Fig. 1). Among interesting results, Jermyn[30] finds that at $\mathrm{pH}>7$, the hydrolysis of both $\delta$ - and $\gamma$-gluconolactones is pseudo first order in a buffer that maintains a constant $\mathrm{pH}$ (see Fig. 7 in Ref. [30]). In contrast, at $\mathrm{pH}<7$, this author assumed 
a negligible direct hydrolysis of $\gamma$-GL, which implies an isomerisation step into $\delta$-GL (analogous to the "backward" isomerisation reaction with constant $k_{m}$ in Fig. 1) followed by the hydrolysis of $\delta$-GL into gluconic acid. Alternatively, in [31] the authors concluded to a reversible $\gamma$ - to $\delta$ - conversion, with hydrolysis of both $\delta$ - and $\gamma$-GL. Later, these authors revised their theory[32] and concluded that $\gamma$ - to $\delta$-GL are in equilibrium, whereas only the $\delta$-GL undergoes hydrolysis. This brief review of studies on 6 -gluconolactones illustrates the dependence on the experimental conditions of a kinetic scheme similar to the reaction pathway depicted in Fig. 1. Such a variability is also very likely to be observed for the case of 6-phosphogluconolactones (PGLs). For the case at hand, where experiments are performed in pseudo in-vivo conditions, data strongly support the assumption of a reversible isomerisation, in agreement with the previous findings of Ref.[2]. In conclusion, we have demonstrated that DDNP experiments provide experimental observations that can be used to extract kinetics parameters of a complex chemical reaction pathway on time scales of a few seconds, which are hardly accessible by other means. This study also points to some specificities of data analysis using measurements with such high sensitivity, where a very high dynamic range allows to quantitatively analyze data that are otherwise unobservable.

\section{References}

[1] V. Soskic, K. Groebe, A. Schrattenholz, Exp. Gerontol. 2008, 43, 247-257.

[2] E. Miclet, V. Stoven, P. A. M. Michels, F. R. Opperdoes, J.-Y. Lallemand, F. Duffieux, J. Biol. Chem. 2001, 276, $34840-34846$.

[3] M. Y. Galperin, O. V. Moroz, K. S. Wilson, A. G. Murzin, Mol. Microbiol. 2006, 59, 5-19.

[4] Y. She, X. Xu, A. Yakunin, S. Dhe-Paganon, L. Donald, K. Standing, D. Lee, Z. Jia, T. Cyr, J. Proteome Res. 2010, $\underline{9}, 3311-3318$.

[5] S. Bowen, C. Hilty, Angew. Chem. Int. Ed. 2008, 47, 5235 -5237.

[6] A. Barb, S. Hekmatyar, J. Glushka, J. Prestegard, J. Magn. Res. 2010, 228, 59-65.

[7] H. Allouche-Arnon, Y. Hovav, L. Friesen-Waldner, J. Sosna, J. M. Gomori, S. Vega, R. Katz-Brull, NMR Biomed. 2014, 27, 656-662.

[8] E. Miclet, D. Abergel, A. Bornet, J. Milani, S. Jannin, G. Bodenhausen, J. Phys. Chem. Lett. 2014, $\underline{5}$, $3290-3295$.

[9] S. Jannin, A. Bornet, S. Colombo, G. Bodenhausen, Chem. Phys. Lett. 2011, 517, 234-236.

[10] P. A. Srere, Science 1967, $\underline{158}, 936-937$.

[11] K. R. Albe, M. H. Butler, B. E. Wright, J. theor. Biol. 1990, 143, 163-195.

[12] M. H. Lerche, P. R. Jensen, M. Karlsson, S. Meier, Analytical Chemistry 2014, 87, 119-132.

[13] M. Karlsson, P. R. Jensen, J. O. Duus, S. Meier, M. H. Lerche, Appl Magn Reson 2012, 43, 223-236.

[14] H. Allouche-Arnon, M. H. Lerche, M. Karlsson, R. E. Lenkinski, R. Katz-Brull, Contrast Media Mol. Imaging 2011, 6, 499-506.

[15] S. Meier, M. Karlsson, P. R. Jensen, M. H. Lerche, J. Ø. Duus, Molecular Biosystems 2011, $\underline{7}, 2834-2836$.

[16] S. Meier, P. R. Jensen, J. Ø. Duus, FEBS Letters 2011, 585, 3133-3138. 
[17] S. Meier, P. R. Jensen, J. Ø. Duus, ChemBioChem 2012, 13, 308-310.

[18] S. Meier, N. Solodovnikova, P. R. Jensen, J. Wendland, ChemBioChem 2012, 13, 2265-2269.

[19] T. Harris, H. Degani, L. Frydman, NMR in Biomedicine 2013.

[20] C. E. Christensen, M. Karlsson, J. R. Winther, P. R. Jensen, M. H. Lerche, The Journal of Biological Chemistry 2014, 289, 2344-2352.

[21] M. H. Lerche, P. R. Jensen, M. Karlsson, S. Meier, Chem. Eur. J. 2013, 19, 13288-13293.

[22] M. Salas, E. Vinuela, A. Sols, J. Biol. Chem. 1965, 240, 561-586.

[23] J. M. Bailey, P. H. Fishman, P. G. Pentchev, Journal of Biological Chemistry 1968, 243, 4827-4831.

[24] J. M. Bailey, P. H. Fishman, P. G. Pentchev, Biochemistry 1970, $\underline{9}$, 1189-1194.

[25] E. M. Chance, B. Hess, T. Plesser, B. Wurster, Eur. J. Biochem. 1975, 50, 419-424.

[26] Scilab, a free scientific software package, Copyright 1989-2005. INRIA ENPC,www.scilab.org.

[27] R. Storn, K. Price, J. Global Optim. 1997, 11, 341-359.

[28] W. D. Carlo, H. Jarausch, http://www1.icsi.berkeley.edu/ storn/Journal of Global Optimization.

[29] E. T. Rakitzis, P. Papandreou, Chem.-Biol. Interact. (1998) 1998, 113, 205-216.

[30] M. Jermyn, BBA 1960, 37, 78-92.

[31] T. Takahashi, M. Mitsumoto, Nature 1963, 199, 765-767.

[32] K. Shimahara, T. Takahashi, BBA 1970, 201, 410-415. 


\section{Graphical abstract}

High sensitivity in NMR: Dissolution Dynamic Nuclear Polarization (DDNP) can be used to monitor complex and fast chemical reactions. A careful quantitative analysis of NMR signals is performed allowing for the determination of kinetic constants of reactions occurring in the Pentose Phosphate Pathway

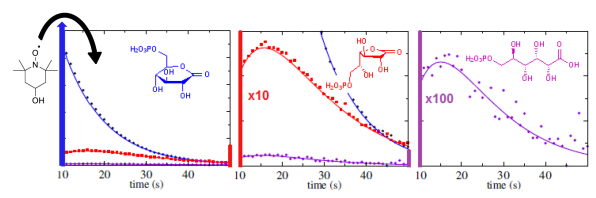

